\title{
Magnetic holes in plasmas close to the mirror instability
}

\author{
D. Borgogno, T. Passot, and P. L. Sulem \\ CNRS, Observatoire de la Côte d'Azur, B.P. 4229, 06304 Nice Cedex 4, France
}

Received: 8 May 2007 - Revised: 27 June 2007 - Accepted: 27 June 2007 - Published: 9 July 2007

\begin{abstract}
Non-propagating magnetic hole solutions in anisotropic plasmas near the mirror instability threshold are investigated in numerical simulations of a fluid model that incorporates linear Landau damping and finite Larmor radius corrections calculated in the gyrokinetic approximation. This FLR-Landau fluid model reproduces the subcritical mirror bifurcation recently identified on the Vlasov-Maxwell system, both by theory and numerics. Stable magnetic hole solutions that display a polarization different from that of HallMHD solitons are indeed obtained slighlty below threshold, while magnetic patterns and spatio-temporal chaos emerge when the system is maintained in a mirror unstable regime.
\end{abstract}

\section{Introduction}

Many observations both in planetary magnetosheaths and in the solar wind indicate the presence of magnetic increases and decreases, either periodic or in isolation, that have commonly been attributed to nonlinearly saturated mirror modes (Kaufmann et al., 1970; Sperveslage et al. , 2000; Joy et al., 2006). These magnetic fluctuations that are quasi-stationary in the plasma frame, are observed in relatively high $\beta$ environments, in anticorrelation with pressure and density. Moreover, their shape is cigar-like, elongated along a direction making a small angle with the ambient magnetic field (Lucek et al., 2001). In many cases, trains of quasi-monochromatic mirror modes are seen, that are nevertheless not perfectly symmetric, their degree of skewness, that measures preference towards magnetic holes or humps, displaying a clear statistical correlation with distance to the mirror instability threshold (Génot et al., 2006). Slighlty above onset, the fluctuations are quasi-sinusoidal. Further above onset (i.e. often for larger values of $\beta$ ), magnetic humps are preferably

Correspondence to: T. Passot

(passot@obs-nice.fr) observed. Interestingly, mirror structures in the form of finite amplitude magnetic holes are also present both above and below the mirror threshold, indicating the existence of a bistable regime.

Several numerical and analytical works have been devoted to the mirror instability, mostly in the linear regime (Vedenov and Sagdeev, 1958; Hall, 1979; Gary, 1992; Southwood and Kivelson, 1993; Pokhotelov et al., 2002). The nonlinear saturation remains in contrast an unsettled issue. When the distance to threshold and the initial data are small, quasilinear effects may have time to significantly affect the bulk of the distribution function if the system is sufficiently homogeneous, (Shapiro and Shevchenko, 1964). When the linear growth is capable of producing significant magnetic field distortions, the hydrodynamic nonlinearity can overcome nonlinear kinetic effects. Recently, a systematic reductive perturbative expansion of the Vlasov-Maxwell equations close to the mirror instability threshold has been carried out (Kuznetsov et al., 2007). The resulting equation where linear Landau damping and finite Larmor radius corrections are the only relevant kinetic effects, possesses a finite-time singularity, indicating that the physical system rapidly leaves the domain of validity of the asymptotics. In particular, this singularity is a possible signature of a subcritical bifurcation and of the existence of finite amplitude solutions, even close to the instability threshold, making the nonlinear saturation not amenable to a rigorous perturbative treatment.

A few phenomenological models have attempted to address the nonlinear saturation of the mirror instability (Kivelson and Southwood, 1996; Pantellini, 1998), based on the cooling of trapped particles in magnetic troughs. They mostly predict the formation of deep magnetic holes with magnetic humps occuring only for large values of $\beta$, but do not mention the possible existence of bistability, in contrast with observational evidences.

An alternative scenario based on a dominant saturating effect originating from the local variation of the ion Larmor

Published by Copernicus Publications on behalf of the European Geosciences Union and the American Geophysical Union. 
radius was recently suggested (Kuznetsov et al., 2007). In regions of weaker magnetic field and larger perpendicular temperature, the ion Larmor radius is indeed larger, making the stabilizing effect of finite Larmor radius (FLR) corrections more efficient than within the linear description. As a result, the mirror instability will be more easily quenched in magnetic minima than in maxima, making magnetic humps more likely to form in the saturation phase of the linear mirror instability. The variation of the local ion Larmor radius can be phenomenologically supplemented to the asymptotic equation mentioned above, and the resulting model indeed leads to the formation of magnetic humps above threshold, a behavior similarly displayed by direct numerical simulations of the Vlasov-Maxwell equations (Califano et al., 2007 ${ }^{1}$ ). A bistable region below the mirror instability threshold was also identified both within this phenomenological model and in direct simulations of the Vlasov-Maxwell equations. Starting with finite amplitude magnetic perturbations in the form of localized holes, the system rapidly stabilizes on stationary solutions. Existence of such structures is compatible with satellite observations. Note that with anisotropic MHD supplemented by an equation of state appropriate for the quasistatic regime (Passot and Sulem, 2006a) and thus capable of accurately reproducing the mirror instability threshold (see also Hau et al., 2005), an energy minimization principle can capture bistability, as well as the preference of magnetic humps at larger values of $\beta$ and/or for a larger distance from threshold (Passot et al., 2006), indicating that fluid models retain essential ingredients associated with the mirror bifurcation structure.

Anisotropic MHD models including linear Landau damping as the only kinetic effect (Snyder et al., 1997), reproduce the mirror instability growth rate at large scale, but can hardly address the nonlinear dynamics since in this quasihydrodynamic regime the instability growth rate increases linearly with the perturbation wavenumber. Attempts based on usual anisotropic MHD supplemented with an exponential filtering of the small scales (Baumgärtel, 2001) show the formation of magnetic structures that subsist below the instability threshold. Their properties and shape are however not in agreement with observations. More recently, a FLRLandau fluid model was derived that correctly captures the mirror instability growth rate at all scales, by incorporating linear Landau damping and FLR terms calculated within the gyrokinetic approximation, in a fluid hierarchy closed at the level of the pressure tensor (Passot and Sulem, 2006a) or of the fourth order moments (Sulem and Passot, 2007; Passot and Sulem, 2007). This model was integrated in mirror unstable regimes, showing the formation of sharp magnetic holes and transient humps at larger values of $\beta$ (Passot and Sulem, 2006b). During the saturation phase, mean temper-

\footnotetext{
${ }^{1}$ Califano, F., Hellinger, P., Kuznetsov, E., Passot, T., Sulem, P. L., and Travnicek, P.: Nonlinear mirror mode dynamics: simulations and modeling, preprint, 2007.
}

atures rapidly evolve in a way as to reduce the distance to threshold. The structures that are formed are not perfectly stationary, their amplitude decreasing on a longer time scale. This tendency is probably due to the lack of particle trapping. This model does not yet incorporate the local variation of the Larmor radius and is thus not capable of reproducing the formation of magnetic humps as the development of the mirror instability from random initial noise, at moderate distance from threshold.

The goal of this paper is to investigate the properties of mirror structures in a parameter regime close to the mirror threshold, paying particular attention to the bistable regime correctly captured by the FLR-Landau fluid model. For this purpose, initial conditions will be taken in the form of large amplitude magnetic fluctuations and mean temperatures will be kept fixed in order to permit a truely stationary regime. In Sect. 2, the FLR-Landau fluid model is briefly described and tested against kinetic theory in the linear regime. A simulation starting from random initial conditions in close proximity to threshold is discussed in Sect. 3 and compared with predictions of the asymptotic theory developed in Kuznetsov et al. (2007). Section 4 is devoted to the investigation of solutions obtained when fixing mean ion pressures, both above threshold, where magnetic patterns are observed, and below threshold where a bistable regime is found. Section 5 briefly addresses regimes arising when the angle between the ambient magnetic field and the wavevector is decreased, leading to a transition between non-propagating and slowly propagating mirror structures. In the latter case, the amplitude of the magnetic hole remains almost constant over very long times, even when the mean temperatures can freely evolve in time. Section 6 is a brief conclusion.

\section{The FLR-Landau fluid model}

As a consequence of their quasi-transverse and nonpropagating character, mirror modes near threshold obey a slow dynamics involving small transverse scales and making FLR effects relevant. The fluid approach developed for addressing slow-dynamics phenomena in fusion plasmas, known as "gyro-fluids" (Brizard, 1992; Dorland and Hammett, 1993; Beer and Hammet, 1996), is obtained by taking velocity moments of the gyrokinetic equation (Howes et al, 2006). Nonlinear FLR corrections to all orders are captured, but a closure of the hierarchy is still required. Furthermore, the equations are not written in the physical coordinates but in the gyrocenter variables. A simpler description retaining hydrodynamic nonlinearities together with a linear approximation of FLR contributions estimated from the kinetic theory assuming the gyrokinetic scaling, was initiated by Smolyakov et al. (1995), in the limit where Landau damping is negligible. Based on this idea, we have recently developed a generalization of the Landau fluid approach, by deriving equations for the hydrodynamic moments from 
the Vlasov-Maxwell system (Passot and Sulem, 2007) rather than from the drift-kinetic equation (Snyder et al., 1997). In addition to the hierarchy closure, this approach involves the modeling of FLR effects that is carried out by expressing the non-gyrotropic part of tensors such as pressures, heat fluxes, etc. in terms of lower-rank moments, in a way consistent with the linear kinetic theory in the low-frequency limit $\epsilon \sim \omega / \Omega_{i} \ll 1$, for both quasi-transverse fluctuations $\left(k_{\|} / k_{\perp} \sim \epsilon\right)$ with no condition on $k_{\perp} r_{L}$ (as in gyrokinetic and gyrofluid approaches), but also for hydrodynamic scales with $k_{\|} \sim k_{\perp} \ll 1 / r_{L}$. Here $\Omega_{i}$ denotes the ion cyclotron frequency and $r_{L}$ the ion Larmor radius. Accurate simulations of scales comparable to a fraction of the ion inertial length indeed requires to retain much smaller transverse scales. Note that at large scales, the model, which then reduces to usual anisotropic MHD, also captures the fast waves.

The resulting FLR-Landau fluid model is integrated in one space dimension, assuming that all the fields only depend on a coordinate $\xi$, along a direction of the $(x, z)$-plane making an angle $\alpha$ with the z-axis defined by the uniform ambient magnetic field (of magnitude $B_{0}$ ). The total plasma density field is normalized by $\rho_{0}$, the magnetic field by $B_{0}$, the velocities by the Alfvén velocity $v_{A}=B_{0} /\left(4 \pi \rho_{0}\right)^{1 / 2}$, the pressures by the parallel ion pressure $p_{0}=p_{\|}^{i(0)}$, the heat fluxes by $p_{0} v_{A}$ and the fourth rank moments by $p_{0} v_{A}^{2}$. With the unit of length $L$, one defines the dispersion parameter $R_{i}=L^{-1} v_{A} / \Omega_{i}$ which, in all subsequent simulations, will be taken equal to unity. The parameter $\beta=8 \pi p_{0} / B_{0}^{2}$ measures the ratio of the (parallel) thermal to the magnetic pressure. Velocities without superscripts refer to the ion velocity. The electron velocity $u^{e}$ is given by

$$
\begin{aligned}
& u_{x}^{e}=u_{x}+R_{i} \cos \alpha \frac{\partial_{\xi} b_{y}}{\rho} \\
& u_{y}^{e}=u_{y}-R_{i} \frac{\partial_{\xi} b_{p}}{\rho} \\
& u_{z}^{e}=u_{z}-R_{i} \sin \alpha \frac{\partial \xi b_{y}}{\rho},
\end{aligned}
$$

where we define $b_{p}=\cos \alpha \quad b_{x}-\sin \alpha \quad b_{z}$. We also define $\bar{u}=\sin \alpha u_{x}+\cos \alpha u_{z}$ which, together with $\nabla \cdot u=\partial_{\xi} \bar{u}$, take the same form when using the electron velocity. When integrated, the divergenceless condition $\nabla \cdot b=0$ rewrites $\cos \alpha b_{z}+\sin \alpha \quad b_{x}=\cos \alpha$, which allows to write $\nabla \cdot \widehat{b}=\nabla \cdot$ $(b /|b|)=-\cos \alpha \partial_{\xi}|b| /|b|^{2}$.

The model involves dynamical equations for the ion density $\rho$ and velocity $u$, the magnetic field components $b_{p}$ and $b_{y}$, together with, for each species $r$, the gyrotropic parallel and perpendicular pressures $p_{\|}^{r}$ and $p_{\perp}^{r}$, and the heat fluxes $q_{\|}^{r}$ and $q_{\perp}^{r}$. They read

$$
\begin{aligned}
& \partial_{t} \rho+\partial_{\xi}(\rho \bar{u})=0 \\
& \partial_{t}\left(\rho u_{j}\right)+\partial_{\xi}\left(F_{j}^{1}+F_{j}^{2}\right)=0 \\
& \partial_{t} b_{p}=\partial_{\xi} E_{y} \\
& \partial_{t} b_{y}=-\partial_{\xi} E_{p}
\end{aligned}
$$

$$
\begin{aligned}
\partial_{t} p_{\|=}^{r}= & \partial_{\xi}\left(\bar{u} p_{\|}^{r}+\frac{\cos \alpha}{|b|} q_{\|}^{r}+\sin \alpha S_{x}^{\| r}\right) \\
& +2 q_{\perp}^{r} \nabla \cdot \widehat{b}-2 p_{\|}^{r} \widehat{b} \cdot \nabla u^{r} \cdot \widehat{b} \\
\partial_{t} p_{\perp}^{r}= & -\partial_{\xi}\left(\bar{u} p_{\perp}^{r}+\frac{\cos \alpha}{|b|} q_{\perp}^{r}+\sin \alpha S_{x}^{\perp r}\right) \\
& -q_{\perp}^{r} \nabla \cdot \widehat{b}-p_{\perp}^{r} \nabla \cdot u+p_{\perp}^{r} \widehat{b} \cdot \nabla u^{r} \cdot \widehat{b} \\
\partial_{t} q_{\|}^{r}= & -\partial_{\xi}\left(\bar{u} q_{\|}^{r}+\frac{\cos \alpha}{|b|} \tilde{r}_{\|\|}^{r}\right)-\frac{3 \beta}{2} p_{\|}^{r} \frac{\cos \alpha}{|b|} \partial_{\xi}\left(\frac{p_{\|}^{r}}{\rho^{r}}\right) \\
& -3 q_{\|}^{r} \widehat{b} \cdot \nabla u^{r} \cdot \widehat{b}+3 \tilde{r}_{\| \perp}^{r} \nabla \cdot \widehat{b} \\
\partial_{t} q_{\perp}^{r}= & -\partial_{\xi}\left(\bar{u} q_{\perp}^{r}+\frac{\cos \alpha}{|b|} \tilde{r}_{\| \perp}^{r}\right)-\frac{\beta}{2} p_{\|}^{r} \frac{\cos \alpha}{|b|} \partial_{\xi}\left(\frac{p_{\perp}^{r}}{\rho^{r}}\right) \\
& -q_{\perp}^{r} \nabla \cdot u-\left(\frac{\beta p_{\perp}^{r}}{2 \rho^{r}}\left(p_{\|}^{r}-p_{\perp}^{r}\right)+\tilde{r}_{\| \perp}^{r}-\tilde{r}_{\perp \perp}^{r}\right) \nabla \cdot \widehat{b} \\
& +\cos \alpha \partial_{\xi} R_{N G}^{r} \cdot
\end{aligned}
$$

The equations for the gyrotropic fourth rank cumulants $\tilde{r}_{\|\|}^{r}$, $\tilde{r}_{\| \perp}^{r}$ and $\tilde{r}_{\perp \perp}^{r}$ are given in Eqs. (A12)-(A16). The electric field and flux terms entering the above equations are given by

$$
\begin{aligned}
& E_{p}=\cos \alpha E_{x}-\sin \alpha E_{z} \\
& E_{j}=-(u \times b)_{j}-\frac{R_{i}}{\rho} \partial_{\xi} F_{j}^{2} \\
& F_{x}^{1}=\rho \bar{u} u_{x}+\frac{\beta}{2}\left(\sin \alpha p_{\perp}^{i}+\cos \alpha\left(p_{\|}^{i}-p_{\perp}^{i}\right) \frac{b_{x}}{|b|^{2}}\right. \\
& \left.+\sin \alpha \Pi_{x x}+\cos \alpha \Pi_{x z}\right) \\
& F_{y}^{1}=\rho \bar{u} u_{y}+\frac{\beta}{2}\left(\cos \alpha\left(p_{\|}^{i}-p_{\perp}^{i}\right) \frac{b_{y}}{|b|^{2}}\right. \\
& \left.+\sin \alpha \Pi_{x y}+\cos \alpha \Pi_{y z}\right) \\
& F_{z}^{1}=\rho \bar{u} u_{z}+\frac{\beta}{2}\left(\cos \alpha p_{\perp}^{i}+\cos \alpha\left(p_{\|}^{i}-p_{\perp}^{i}\right) \frac{b_{z}}{|b|^{2}}\right. \\
& \left.+\sin \alpha \Pi_{x z}\right) \\
& F_{x}^{2}=\sin \alpha \frac{|b|^{2}}{2}-\cos \alpha b_{x}+\frac{\beta}{2}\left(\sin \alpha p_{\perp}^{e}\right. \\
& \left.+\cos \alpha\left(p_{\|}^{e}-p_{\perp}^{e}\right) \frac{b_{x}}{|b|^{2}}\right) \\
& F_{y}^{2}=-\cos \alpha b_{y}+\frac{\beta}{2}\left(\cos \alpha\left(p_{\|}^{e}-p_{\perp}^{e}\right) \frac{b_{y}}{|b|^{2}}\right) \\
& F_{z}^{2}=\cos \alpha \frac{|b|^{2}}{2}-\cos \alpha b_{z}+\frac{\beta}{2}\left(\cos \alpha p_{\perp}^{e}\right. \\
& \left.+\cos \alpha\left(p_{\|}^{e}-p_{\perp}^{e}\right) \frac{b_{z}}{|b|^{2}}\right) .
\end{aligned}
$$

The specific form of the gyroviscous tensor $\Pi$ and of the nongyrotropic contributions to the fourth-rank cumulant $R_{N G}^{r}$, as well as the transverse components of the fluxes of parallel and perpendicular heat $S_{x}^{\| r}$ and $S_{x}^{\perp r}$ are computed from the linear kinetic theory and given in the Appendix. The work 


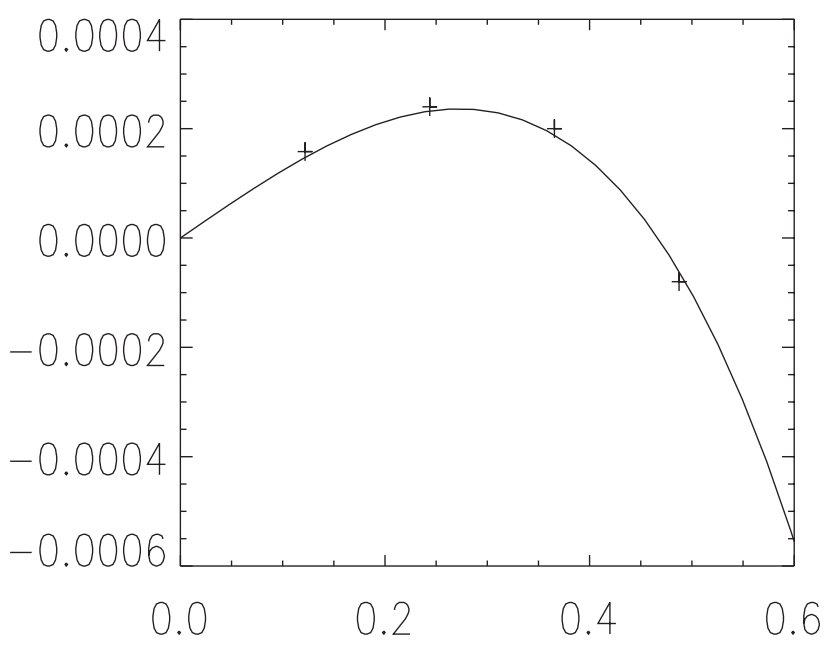

Fig. 1. Normalized growth rate $\Im(\omega) / \Omega_{i}$ versus $k_{\perp} r_{L}$ for mirror modes with $\beta=5, \tau=0.1, \alpha=\cos ^{-1}(0.1), T_{\perp i} / T_{\| i}=1.2$ and $T_{\perp e} / T_{\| e}=1$.

done by the gyroviscous force (see Eqs. 8 and 9 in Goswami et al., 2005) is not included in the pressure equations because it originates from nonlinear terms involving FLR corrections, a type of contributions that have been systematically neglected. Retaining among them, those contributing to the conservation of the total energy

$E=\int\left[\frac{\rho u^{2}}{2}+\frac{b^{2}}{2}+\frac{\beta}{2}\left(p_{\perp i}+p_{\perp e}+\frac{1}{2}\left(p_{\| i}+p_{\| e}\right)\right)\right] d \xi$

is possible. We nevertheless choose to neglect these contributions in order to estimate the accuracy of the above approximations. It turns out that in the forthcoming simulations $E$ does not vary by more than one percent.

The above equations are integrated numerically in a periodic domain of size $2 \pi D$ in units of ion inertial length, using a pseudo-spectral scheme based on fast Fourier transforms. Derivatives, Hilbert transforms and operators involving the $\Gamma_{v}$ 's (see Appendix) are calculated in Fourier space where they just reduce to multiplications. Nonlinearities are evaluated in real space. At each time step, and for each field, Fourier modes are truncated at $k_{\max } / 2$, which does not amount to a complete dealiasing since the nonlinearities are not only of cubic type but also involve divisions. Time stepping is performed with a second order Adams-Bashforth scheme. No additional dissipation nor filtering is introduced. Several linear tests of the model and of the code have been performed (Passot and Sulem, 2007), and in particular, the growth rate of the mirror instability is accurately reproduced. Figure 1 displays a comparison of the mirror growth rate given by linear kinetic theory using the WHAMP code (Rönnmark, 1983) (solid line) with the growth rate measured on a short-time integration of the FLR-
Landau fluid equations initialized with a small amplitude random noise superimposed on the equilibrium state characterized by $\beta=5, \alpha=\cos ^{-1}(0.1), T_{\| e}=T_{\| i}, T_{\perp i} / T_{\| i}=1.2$ and $T_{\perp e} / T_{\| e}=1$. With these parameters for which the growth rate is rather small compared with the ion cyclotron frequency and the angle $\alpha$ in a range where the gyrokinetic ordering applies, the agreement is excellent. At larger deviations from threshold, a small discrepency develops near the maximum growth rate (Passot and Sulem, 2007).

\section{Mirror dynamics very close to threshold}

In order to investigate the nonlinear development of the mirror instability very close to threshold, an initial state was selected with $\beta=5, \alpha=88.85^{\circ}, T_{\perp i} / T_{\| i}=1.18$ and $T_{\perp e}=$ $T_{\| e}=0.05 T_{\| i}$, for which the maximum growth rate is about $9.5 \times 10^{-6} \Omega_{i}$. Starting with random initial noise of amplitude $10^{-4}$ superimposed on a uniform density and longitudinal magnetic field, we first observe the formation of a growing quasi-sinusoidal wave whose wavelength is about $94 l_{i}$, consistent with the predicted most unstable wavenumber, given by $k_{\perp} r_{L}=0.16$ (Fig. 2a). This scale is large enough compared with the ion gyroradius for the dynamics to be described by the asymptotic equation derived in Kuznetsov et al. (2007). After the linear regime, the dynamics develops an acceleration phase that manifests itself by a super-exponential growth of mode 1 between times $t=0.55 \times 10^{6} \Omega_{i}^{-1}$ and $t=0.78 \times 10^{6} \Omega_{i}^{-1}$. The solution displays at this time a magnetic hole whose depth is about $28 \%$ (Fig. 2b), a large amplitude consistent with the singular behavior of the asymptotic solution. The FLR-Landau fluid model, that includes richer nonlinearities than the asymptotic equation, is capable of arresting the singularity. The solution then relaxes towards the uniform state (Fig. 2c) on a time scale of the order of $10^{5} \Omega_{i}^{-1}$. The corresponding variation of the mean temperatures that evolve towards a regime where the system is mirror stable, are displayed in Fig. 3. It is interesting to note that when preserving close proximity to threshold, but considering variation in a more oblique direction, no quasi-singular dynamics is observed, the system saturating smoothly on small-amplitude quasi-sinusoidal mirror waves.

Quasi-linear effects (Shapiro and Shevchenko, 1964) which, in Vlasov-Maxwell simulations, are observed to operate at early time and in large domains, are not contained in the the FLR-Landau fluid model. The latter model, like the asymptotic equation, however captures a branch of solutions that is observed on Vlasov simulations for larger distance from threshold or at later times in the evolution (Califano et al., $2007^{1}$ ). It thus not only correctly reproduces the linear dynamics of the mirror modes, but also incorporates the ingredients of a subcritical bifurcation, leading to the development of large amplitude structures. 

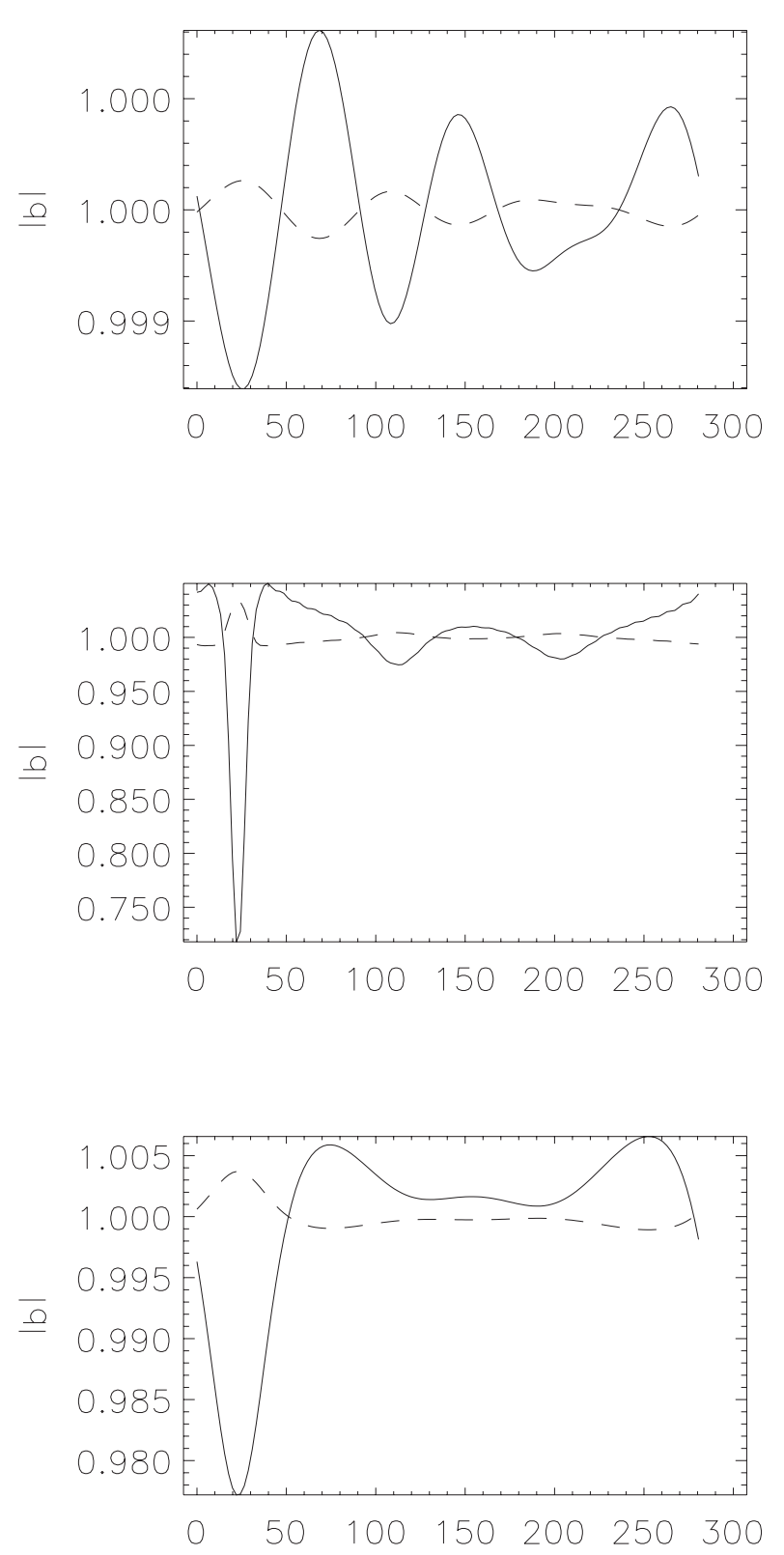

Fig. 2. Snapshots of a simulation illustrating the development of the mirror instability very close to threshold, with $\beta=5, \alpha=88.85^{\circ}$, $T_{\perp i} / T_{\| i}=1.18$ and $T_{\perp e}=T_{\| e}=0.05 T_{\| i}$ at times $t=350 \times 10^{3} \Omega_{i}^{-1}$, $t=780 \times 10^{3} \Omega_{i}^{-1}$ and $t=850 \times 10^{3} \Omega_{i}^{-1}$. Displayed are the magnetic field amplitude (solid line) and the density (dashed line) as a function of space in units of ion inertial length $l_{i}=\frac{v_{A}}{\Omega_{i}}$.

\section{Nonlinear structures with constant mean ion pres- sures}

Since in the simulation discussed in Sect. 3, as well as in those reported in Passot and Sulem (2006b), the structures that form as a result of the mirror instability are not per-
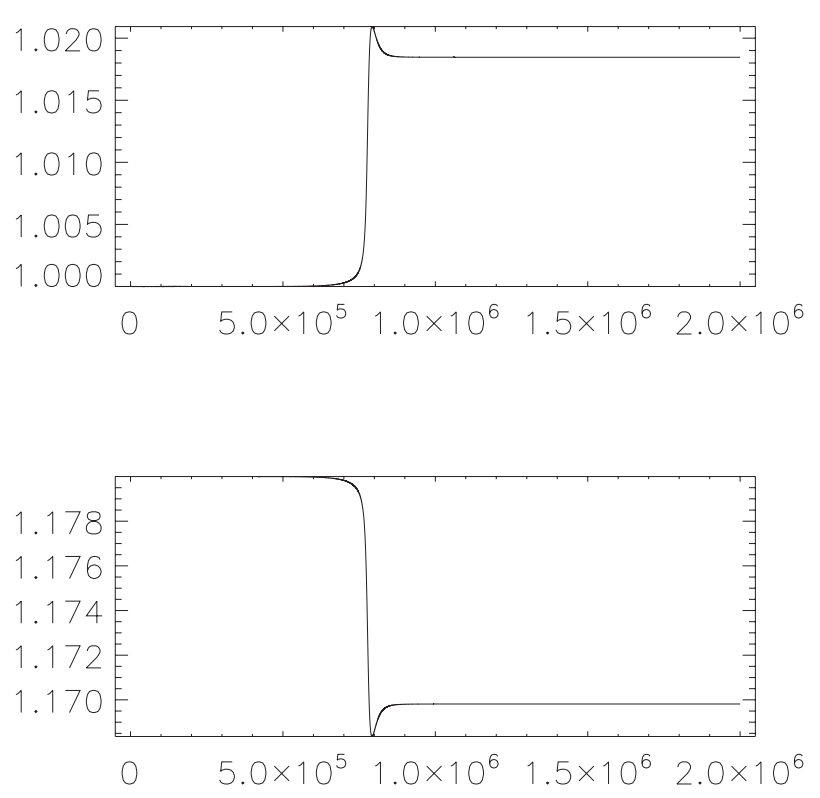

Fig. 3. Evolution of the parallel (top) and perpendicular (bottom) temperatures in the run close to threshold, as a function of time in units of $\Omega_{i}^{-1}$.

fectly stationary but rather slowly relax to the uniform state, it is of interest to enforce stationarity by maintaining constant the mean ion parallel and perpendicular pressures. This is a simple way of imposing a forcing which, in more realistic situations, is obtained through boundary conditions, such as for example an inflow. We shall first address the case above threshold. Any kind of initial condition, either small amplitude noise or finite amplitude perturbations, leads to similar solutions in the form of periodic trains of magnetic holes as depicted in Fig. 4 (top). In this simulation, with $\beta=5, \alpha=78.46^{\circ}, T_{\perp i} / T_{\| i}=1.2$ and $T_{\perp e}=T_{\| e}=0.05 T_{\| i}$, the size of the domain is $94 l_{i}$, so that only two magnetic structures (whose width at mid-height is about $5 r_{L}$ ) can form. As a result, the system is able to reach a stationary regime. The bottom panel of Fig. 4 displays the perpendicular (solid line) and parallel (dashed line) ion temperatures, showing, as for the density (dashed line in the top panel), a clear anticorrelation with the magnetic field amplitude. Note that the parallel temperature presents a sharper profile than the perpendicular one, with a smaller magnitude.

In the case of larger domains, the system cannot reach an equilibrium. With the same plasma parameters as those of Fig. 4 but in a domain four times larger, the dynamics evolves on a slow time scale and involves few Fourier modes. An interesting feature is the transition between the linear phase, dominated by the most unstable mode, and the nonlinearly developed regime where the characteristic scale of the structures is twice smaller than predicted by linear theory, the dominant Fourier mode becoming linearly neutral. 

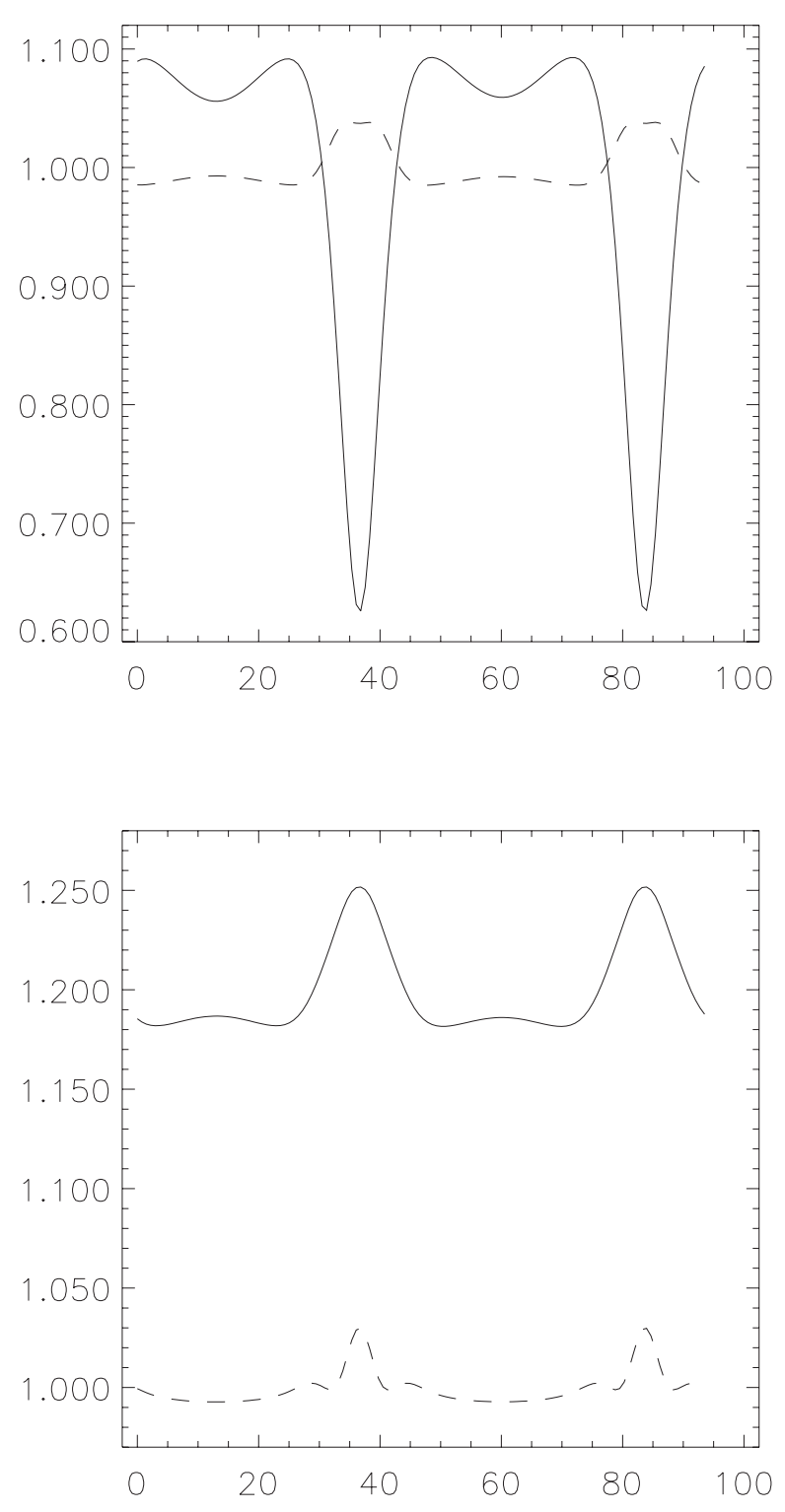

Fig. 4. Stationary solution in a simulation where mean temperatures are kept constant, with $\beta=5, \alpha=78.46^{\circ}, T_{\perp i} / T_{\| i}=1.2$ and $T_{\perp e}=T_{\| e}=0.05 T_{\| i}$. The top panel displays the magnetic field amplitude (solid line) and density (dashed line) as a function of $l_{i}$. The bottom one shows the perpendicular (solid line) and parallel (dashed line) ion temperatures.

At larger distance from threshold, a faster dynamics develops that displays spatio-temporal chaos and involves more Fourier harmonics, most of them corresponding to linearly damped modes, in a way somewhat similar to CLUSTER observations reported by Sahraoui et al. (2006). The spatial Fourier spectrum is however an exponential rather than a power law. Holes form and disappear in a chaotic fashion, and large-scale compression waves are observed to propagate through the domain. Figure 5 displays a snapshot of such a

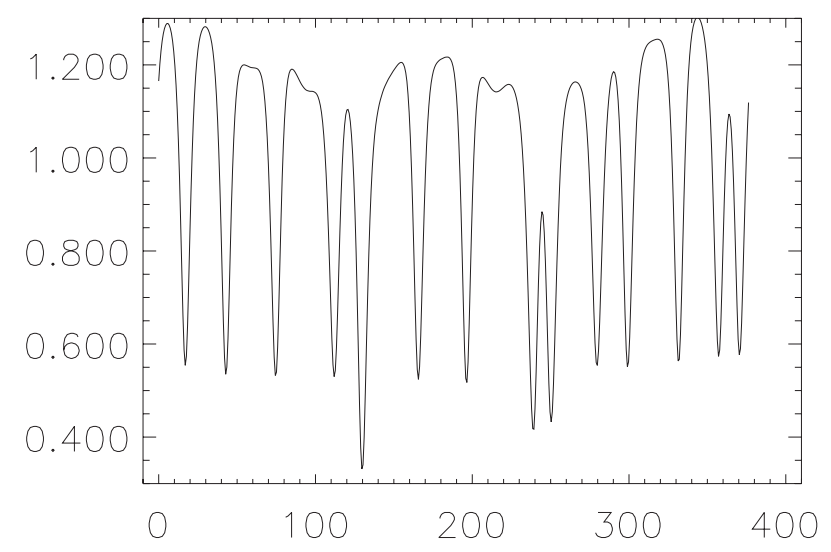

Fig. 5. Snapshot of the magnetic field amplitude in a simulation with fixed mean temperatures in a large domain, displaying spatiotemporal chaos. The parameters are similar to those of Fig. 4 except that $T_{\perp i} / T_{\| i}=1.3$.

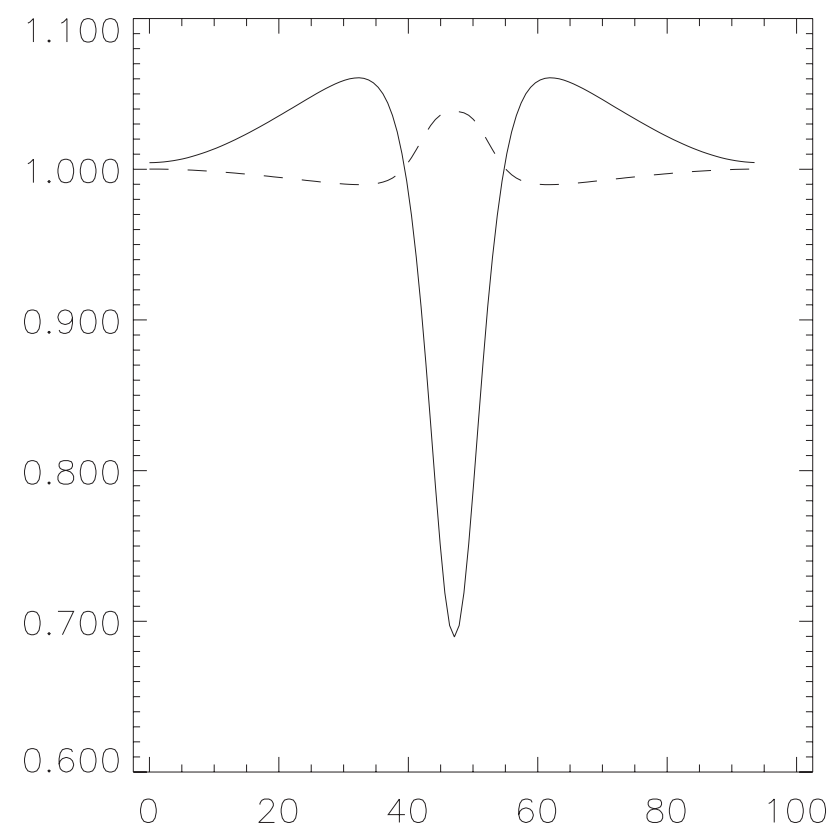

Fig. 6. Magnetic field amplitude (solid line) and density (dashed line) in the stationary regime in a simulation where mean temperatures are kept constant, with $\beta=5, \alpha=78.46^{\circ}, T_{\perp i} / T_{\| i}=1.17$ and $T_{\perp e}=T_{\| e}=0.05 T_{\| i}$.

simulation in the case of an anisotropy $T_{\perp i} / T_{\| i}=1.3$. The general pattern shows an interesting similarity with the hole structures observed in the Jovian magnetosheath (Fig. 1 in Joy et al., 2006).

The saturated equation constructed in Kuznetsov et al. (2007) as well as Vlasov-Maxwell direct simulations (Califano et al., $2007^{1}$ ), indicate that below threshold both the uniform state and isolated magnetic holes are stable solutions. It 
is thus of interest to investigate, in the context of the present fluid model, the range of parameters for which this bistable regime is observed. Since initial conditions in the form of small amplitude noise obviously relax to the uniform state, we here choose to initialize the simulation with a finite size magnetic hole, taking the longitudinal magnetic field in the form $b_{z}=c-0.5 \exp \left[-(x-0.5)^{2} / 0.005\right]$, where $0<\times<1$ is the non-dimensional space variable, and where $c$ is chosen to ensure that $b_{z}$ has mean value 1 . Parameters are identical to those of Fig. 4, except that $T_{\perp i} / T_{\| i}=1.17$, a value for which the uniform state is mirror stable. The hole first adjusts and then stabilizes towards a stationary solution shown in Fig. 6. The hodograph of the magnetic field vector, namely the curve of coordinates $\left(b_{y}, \sin \alpha\left(b_{z}-1\right)-\cos \alpha b_{x}\right)$ is displayed in Fig. 7, showing a small but visible signature of the component of the magnetic field perpendicular to the $\left(\mathbf{k}, \mathbf{B}_{\mathbf{0}}\right)$ plane, also shown in Fig. 8. Interestingly, both the $b_{y}$ and $b_{z}$ components are symmetric with respect to the center of the magnetic hole, making the hodograph fold on itself. This property, also observed in Vlasov simulations (Califano et al., $2007)^{1}$, contrasts with all previous soliton models based on anisotropic Hall-MHD (Stasiewicz, 2004a; Mjølhus, 2006), where $b_{y}$ is found to be antisymmetric. All the velocity components are anti-symmetric, $\bar{u}$ being negligible (about $2 \times 10^{-5}$ ), while $u_{y}$, displayed in Fig. 8 , has a relatively large amplitude with a sharp gradient at the center of the hole, indicating that the gyroviscous tensor plays an important role in the equilibrium. Similar signatures are observed in PIC simulations of non-propagating rarefractive solitary structures generated by particle injection (Baumgärtel et al., 2005) in a plasma that is initially isotropic with a much smaller value of $\beta$ (taken equal to 0.25 ). The heat fluxes also seem to play an important role since no equilibrium can be reached when these terms are removed from the equations. The modeling of these static magnetic holes in terms of soliton solutions of a minimal model (e.g. anisotropic Hall-MHD with leading order FLR corrections) remains an open problem. Their symmetry properties however suggest that they do not correspond to the same branch of solutions as the slow magnetosonic solitons, in contrast with the claim by Stasiewicz (2004b).

It is possible to investigate the subcritical nonlinear solutions in the bistable regime using a continuation procedure taking, for example, the latter solution as initial condition for simulations with the same parameters but smaller anisotropies. Such solutions still exist for $T_{\perp i} / T_{\| i}=1$.16, but seem to disappear at $T_{\perp i} / T_{\| i}=1.15$. In a similar way, it is possible to find subcritical hole solutions with $T_{\perp i} / T_{\| i}=1.17$ and values of $\beta$ down to $\beta=4.6$, corresponding to a slightly larger deviation from threshold. Interestingly, these solutions have a similar magnetic field depression of about $25 \%$ to $30 \%$ (see Fig. 9). The overshoots on the edges of the magnetic holes are almost absent for such subcritical solutions, while they become more prononced above threshold, consistent with CLUSTER observations (Génot et al., 2006).

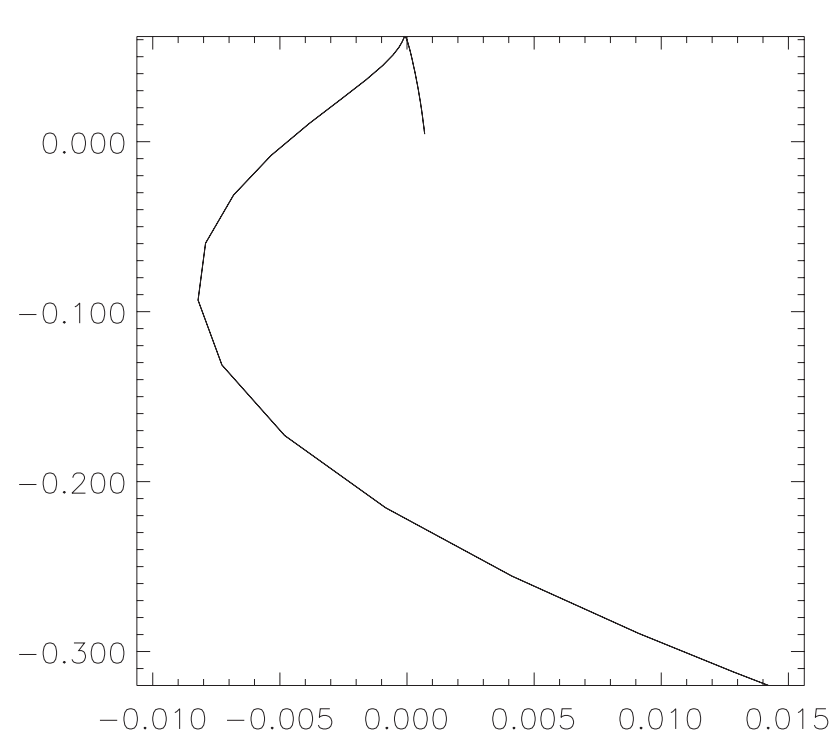

Fig. 7. Hodograph of the magnetic field $\left(b_{y}, \sin \alpha\left(b_{z}-1\right)-\cos \alpha b_{x}\right)$ for the solution displayed in Fig. 6.

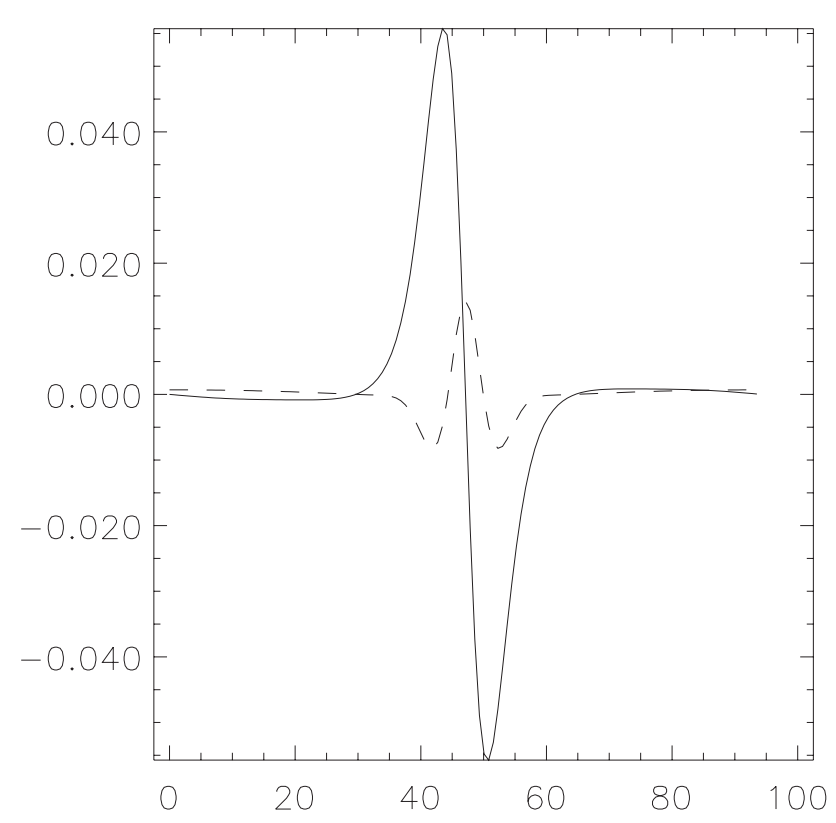

Fig. 8. Profiles of $u_{y}$ (solid) and $b_{y}$ (dashed) for the hole solution displayed in Fig. 6.

If initial conditions in the form of magnetic humps are instead chosen in subcritical situations close to threshold, the system relaxes to the uniform state, as observed in PIC and Eulerian Vlasov simulations (Baumgärtel et al., 2003; Califano et al., 2007 ${ }^{1}$ ). 


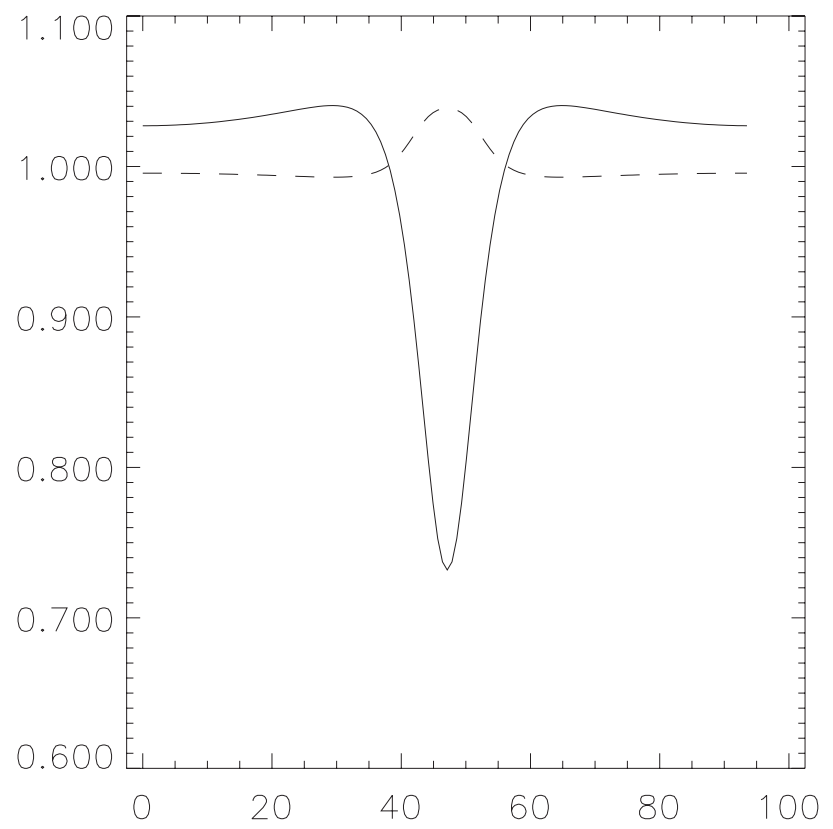

Fig. 9. Magnetic field amplitude (solid line) and density (dashed line) in the stationary regime, in a simulation where mean temperatures are kept constant, with the same parameters as in Fig. 6 except that $\beta=4.6$.

\section{The case of oblique angles}

In this section, we briefly report on the existence of propagating solutions obtained as a result of the nonlinear saturation of the mirror instability developing from small amplitude initial noise at an angle of propagation of $50^{\circ}$ with respect to the ambient magnetic field. The simulations are here performed without fixing the mean temperatures. While for larger angles the structures that form are at rest and slowly relax to the uniform state, for this intermediate propagation angle, slowly propagating solitary-like structures are observed, whose amplitude decreases at a much slower rate. In Fig. 10 are displayed two snapshots at $t=5 \times 10^{4} \Omega_{i}^{-1}$ (right curve) and $t=10^{5} \Omega_{i}^{-1}$ (left curve) of a traveling hole for $\beta=5, T_{\perp i} / T_{\| i}=1.55, T_{\perp e}=T_{\| e}=0.05 T_{\| p}$. For comparison, at a larger angle of propagation, the hole would have completely disappeared after such a long integration time. In the present simulations, the parallel temperature rapidly becomes almost uniform. The profile of the $b_{y}$ magnetic field component presents a small antisymmetric part (not shown).

\section{Conclusions}

We have shown that a fluid model containing linearized Landau damping and FLR effects evaluated in the gyrokinetic approximation is capable of capturing not only the linear dynamics of the mirror instability but also the finite amplitude

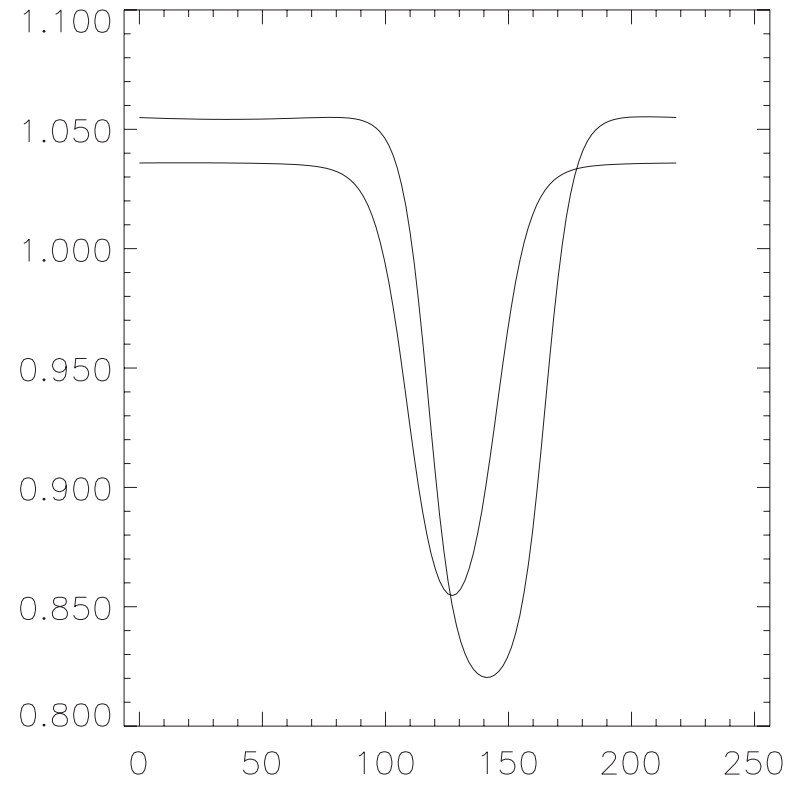

Fig. 10. Two snapshots of the magnetic field amplitude as a function of space in units of $l_{i}$ for a simulation with freely evolving temperatures, with $\beta=5, \alpha=50^{\circ}, T_{\perp i} / T_{\| i}=1.55, T_{\perp e}=T_{\| e}=0.05 T_{\| i}$.

solutions in the form of magnetic holes commonly observed in Vlasov simulations and in satellite data of planetary magnetosheaths. This approach is relevant in cases where nonlinearities originate mainly from hydrodynamic effects. Nonlinear kinetic effects such as particle trapping or nonlinear FLR corrections, not retained by the present description, can however be efficient to saturate the instability. The inclusion of these effects at a phenomenological level in a fluid model is presently an object of investigation. Further work is also needed to clarify the nature of the obtained static magnetic hole solutions, which, in view of their polarization properties, do not appear to belong to the same family as the travelling Hall-MHD solitons.

\section{Appendix}

In the following, the hat symbol denotes the Fourier component of the corresponding fields and the overbar their (time-dependent) space average. The various non-gyrotropic tensor elements are derived in Passot and Sulem (2007) and explicited below in the one-dimensional case.

Gyroviscous stress tensor:

$$
\begin{aligned}
& \Pi_{x x}=-p_{\perp}^{i} \mathcal{F}^{-1}\left(i k \sin \alpha R_{i} \mathcal{A}_{1} \widehat{u}_{y}+\mathcal{A}_{2} \frac{\widehat{T}_{\perp}^{i}}{\bar{T}_{\perp}^{i}}\right) \\
& \Pi_{x y}=p_{\perp}^{i} R_{i} \sin \alpha \partial_{\xi} \mathcal{F}^{-1}\left(\mathcal{B}_{1} \widehat{E}_{y}-\mathcal{B}_{2} \widehat{u}_{x}\right)
\end{aligned}
$$




$$
\begin{aligned}
\Pi_{x z}= & \left(p_{\|}^{i}-p_{\perp}^{i}\right) \mathcal{F}^{-1}\left(\mathcal{C}_{1} \widehat{b}_{x}+i k \sin \alpha R_{i} \mathcal{C}_{2} \widehat{E}_{z}\right) \\
& +\left(2 p_{\|}^{i}-p_{\perp}^{i}\right) \partial_{\xi} \mathcal{F}^{-1}\left(\mathcal{C}_{2} \widehat{E}_{p}\right) \\
\Pi_{y z}= & R_{i} p_{\perp}^{i} \sin \alpha \partial_{\xi} \mathcal{F}^{-1}\left(\mathcal{P}_{y z} \mathcal{D}_{1}\left(\widehat{Q}_{\perp}+\widehat{u}_{z}\right)\right) \\
& +R_{i}\left(2 p_{\|}^{i}-p_{\perp}^{i}\right) \cos \alpha \partial_{\xi} \mathcal{F}^{-1}\left(\mathcal{P}_{y z} \widehat{E}_{y}\right) \\
& +R_{i}\left(p_{\perp}^{i}-p_{\|}^{i}\right) \mathcal{F}^{-1}\left(\mathcal{P}_{y z} \mathcal{D}_{2} \widehat{b}_{y}\right) \\
& +p_{\perp}^{i} R_{i}^{2} \frac{\sin ^{2} \alpha}{\cos \alpha} \partial_{\xi \xi} \mathcal{F}^{-1}(\cos \alpha(-1 \\
& \left.+\frac{\beta}{2}\left(\bar{p}_{\|}^{i}+\bar{p}_{\|}^{e}-\bar{p}_{\perp}^{i}-\bar{p}_{\perp}^{e}\right)\right) \widehat{b}_{y} \\
& \left.+\frac{\beta}{2} \bar{p}_{\perp}^{i}\left(i k R_{i} \sin ^{2} \alpha\right)\left(\mathcal{B}_{1} \widehat{E}_{y}-\mathcal{B}_{2} \widehat{u}_{x}\right)\right) \\
& -\left(\frac{\beta}{4 \pi}\right)^{1 / 2} p_{\|}^{i} k R_{i} \sin \alpha \mathcal{F}^{-1}\left(\psi_{1} \widehat{\rho}+i k R_{i} \sin \alpha \psi_{2} \widehat{u}_{y}\right. \\
& \left.+\widehat{T}_{3} \frac{\widehat{T}_{\perp}^{i}}{\bar{T}_{\perp}^{i}}+\frac{2 i}{\beta k R_{i} \cos \alpha} \psi_{1} \widehat{E}_{z}\right)
\end{aligned}
$$

where

$$
\begin{aligned}
\widehat{Q}_{\perp}= & \frac{\sin \alpha}{\cos \alpha}\left(\mathcal{E}_{1} \widehat{E}_{y}-\left(\mathcal{E}_{3}+1\right) \widehat{u}_{x}+\Gamma_{0} \widehat{E}_{y}\right) \\
& -i\left(\frac{\beta}{\pi}\right)^{1 / 2} \frac{\widehat{T}_{\perp}^{i}}{\bar{T}_{\perp}^{i}}-\left(\frac{\beta}{\pi}\right)^{1 / 2} k R_{i} \sin \alpha \widehat{u}_{y} \\
& -i\left(\frac{\beta}{\pi}\right)^{1 / 2}\left(\frac{\bar{T}_{\perp}^{i}}{\bar{T}_{\|}^{i}}-1\right) \Gamma_{0} \widehat{b}_{z}
\end{aligned}
$$

Transverse fluxes of parallel and transverse heat:

$$
\begin{aligned}
& S_{x}^{\| i}=p_{\|}^{i}\left(p_{\perp}^{i}-p_{\|}^{i}\right) R_{i} \beta \cos \alpha \partial_{\xi} \mathcal{F}^{-1}\left(\mathcal{C}_{2} \widehat{b}_{y}\right) \\
& S_{x}^{\perp i}=p_{\perp}^{i} \mathcal{F}^{-1}\left(\mathcal{E}_{3} \widehat{u}_{x}-\mathcal{E}_{1} \widehat{E}_{y}\right) \\
& S_{x}^{\| e}=-p_{\|}^{e}\left(p_{\perp}^{e}-p_{\|}^{e}\right) R_{i} \beta \cos \alpha \partial_{\xi} b_{y} \\
& S_{x}^{\perp e}=0
\end{aligned}
$$

Non-gyrotropic components of the fourth rank cumulants:

$$
\begin{aligned}
& R_{N G}^{i}=\frac{p_{\perp}^{i}}{\rho}\left(p_{\perp}^{i}-p_{\|}^{i}\right) \mathcal{F}^{-1}\left(-i k \frac{\beta}{2} \cos \alpha \mathcal{R}_{N G 1} \widehat{b}_{z}\right. \\
& \left.+\mathcal{R}_{N G 2} \frac{\widehat{E}_{z}}{R_{i} \bar{T}_{\perp}^{i}}\right)+\frac{p_{\perp}^{i}}{\rho}\left(p_{\perp}^{i}-2 p_{\|}^{i}\right) \times \\
& \mathcal{F}^{-1}\left(\left(\mathcal{C}_{1}+1\right) \frac{\beta}{2} R_{i} k^{2} \sin \alpha \widehat{E}_{p}\right) \\
& R_{N G}^{e}=\frac{p_{\perp}^{e}}{\rho}\left(p_{\perp}^{e}-p_{\|}^{e}\right) \mathcal{F}^{-1}\left(\frac{\beta}{2} \bar{T}_{\perp}^{e} k^{2} R_{i}^{2} \sin ^{2} \alpha \times\right. \\
& \left.\left(-3 \frac{\beta}{2} i k \cos \alpha \widehat{b}_{z}+\frac{\widehat{E}_{z}}{R_{i} \bar{T}_{\perp}^{e}}\right)\right)+\frac{p_{\perp}^{e}}{\rho}\left(p_{\perp}^{e}-2 p_{\|}^{e}\right) \times \\
& \mathcal{F}^{-1}\left(-\frac{\beta}{2} R_{i} k^{2} \sin \alpha \widehat{E}_{p}\right) .
\end{aligned}
$$

Fourth rank cumulants:

$$
\begin{aligned}
& \tilde{r}_{\|\|}^{r}= \mathcal{F}^{-1}\left(\bar{T}_{\|}^{r} \mathcal{R}_{\|\| 1}^{r} \widehat{T}_{\|}^{r}+i \sqrt{\bar{T}_{\|}^{r}} \mathcal{R}_{\|\| 2}^{r} \widehat{q}_{\|}^{r}\right) \\
& \tilde{r}_{\perp \perp}^{i}= \frac{\beta}{2} \bar{T}_{\perp}^{i 2} \mathcal{F}^{-1}\left(\mathcal{R}_{\perp \perp 1}^{i} \widehat{\rho}+i k R_{i} \sin \alpha \mathcal{R}_{\perp \perp 2}^{i} \widehat{u}_{y}\right. \\
&\left.+\mathcal{R}_{\perp \perp 3}^{i} \frac{T_{\perp}^{i}}{\bar{T}_{\perp}^{i}}\right) \\
& \tilde{r}_{\perp \perp}^{e}= 0 \\
&\left(\partial_{t}+\bar{u} \partial_{\xi}\right) \tilde{r}_{\| \perp}^{i}=\mathcal{F}^{-1}\left(-\bar{T}_{\|}^{i} i k \mathcal{R}_{\| \perp 2}^{i} \widehat{q}_{\perp}^{i}-\sqrt{\bar{T}_{\|}^{i}} k \mathcal{R}_{\| \perp 1}^{i} \widehat{\tilde{r}}_{\| \perp}^{i}\right. \\
&\left.\quad+\bar{T}_{\|}^{i} \bar{T}_{\perp}^{i}\left(\bar{T}_{\perp}^{i}-\bar{T}_{\|}^{i}\right) \cos \alpha \sin \alpha k^{2}\left(\mathcal{C}_{1}+1\right) \widehat{b}_{y}\right) \\
&\left(\partial_{t}+\bar{u} \partial_{\xi}\right) \tilde{r}_{\| \perp}^{e}=\mathcal{F}^{-1}\left(-\bar{T}_{\|}^{e} i k \mathcal{R}_{\| \perp 2}^{e} \widehat{q}_{\perp}^{e}-\sqrt{\bar{T}_{\|\|}^{e}} k \mathcal{R}_{\| \perp 1}^{e} \widehat{\tilde{r}}_{\| \perp}^{e}\right. \\
&\left.\quad+\bar{T}_{\|}^{e} \bar{T}_{\perp}^{e}\left(\bar{T}_{\perp}^{e}-\bar{T}_{\|}^{e}\right) \cos \alpha \sin \alpha k^{2} \widehat{b}_{y}\right)
\end{aligned}
$$

The term $\tilde{r}_{\perp \perp}^{i}$ is sometimes filtered at small scales because of the strong increase of its coefficients with the transverse wavenumber. No significant effect results from this approximation, except possibly at the smallest scales.

The various coefficients, functions of the parameter $b \equiv$ $k_{\perp}^{2} r_{L}^{2} / 2=\frac{\beta}{2} \bar{T}_{\perp}^{i} R_{i}^{2} k^{2} \sin ^{2} \alpha$, involve the functions $\Gamma_{0}$ and $\Gamma_{1}$ where $\Gamma_{v}(b)$ is the product of $\exp (-b)$ by the modified Bessel function $I_{v}(b)$. They are given by

$$
\begin{aligned}
& \mathcal{A}_{1}(b)=1-\frac{\Gamma_{1}(b)}{b\left(\Gamma_{0}(b)-\Gamma_{1}(b)\right)}+\frac{\Gamma_{1}(b)}{\Gamma_{0}(b)} \\
& \mathcal{A}_{2}(b)=-\frac{\Gamma_{1}(b)}{\Gamma_{0}(b)} \\
& \mathcal{B}_{1}(b)=\frac{\Gamma_{0}(b)-\Gamma_{1}(b)}{1-\Gamma_{0}(b)}\left(\Gamma_{0}(b)-\Gamma_{1}(b)-\frac{1-\Gamma_{0}(b)}{b}\right) \\
& +\frac{\Gamma_{0}(b)-1-\Gamma_{1}(b)}{b}+2\left(\Gamma_{0}(b)-\Gamma_{1}(b)\right) \\
& \mathcal{B}_{2}(b)=\frac{1}{1-\Gamma_{0}(b)}\left(\Gamma_{0}(b)-\Gamma_{1}(b)-\frac{1-\Gamma_{0}(b)}{b}\right) \\
& \mathcal{C}_{1}(b)=\Gamma_{0}(b)-\Gamma_{1}(b)-1 \\
& \mathcal{C}_{2}(b)=\frac{1-\Gamma_{0}(b)}{b} \\
& \mathcal{D}_{1}(b)=\frac{\Gamma_{0}(b)-\Gamma_{1}(b)}{\Gamma_{0}(b)+b \Gamma_{1}(b)-b \Gamma_{0}(b)-\Gamma_{1}(b) / 2} \\
& \mathcal{D}_{2}(b)=-\mathcal{D}_{1}(b)\left(b\left(\Gamma_{0}(b)-\Gamma_{1}(b)\right)-\Gamma_{0}(b)+1\right) \\
& -\mathcal{C}_{1}(b) \\
& \mathcal{P}_{y z}(b)=\frac{1}{1+b \mathcal{D}_{1}(b)} \\
& \psi_{1}(b)=\Gamma_{1}(b) \mathcal{P}_{y z}(b) \mathcal{D}_{1}(b) \\
& \psi_{2}(b)=-\Gamma_{1}(b) \mathcal{P}_{y z}(b) \mathcal{D}_{1}(b)\left(\frac{1-\Gamma_{0}(b)}{b\left(\Gamma_{0}(b)-\Gamma_{1}(b)\right)}\right. \\
& \left.+1-\frac{2}{\Gamma_{0}(b)}+\frac{\Gamma_{1}(b)}{\Gamma_{0}(b)}\right) \\
& \psi_{3}(b)=\Gamma_{1}(b) \mathcal{P}_{y z}(b) \mathcal{D}_{1}(b)\left(1-\frac{2}{\Gamma_{0}(b)}+\frac{\Gamma_{1}(b)}{\Gamma_{0}(b)}\right)
\end{aligned}
$$




$$
\begin{aligned}
& \mathcal{E}_{1}(b)=\frac{\left(\Gamma_{0}(b)-\Gamma_{1}(b)\right)\left(1-\Gamma_{0}(b)+b \Gamma_{1}(b)-b \Gamma_{0}(b)\right)}{1-\Gamma_{0}(b)} \\
&+2 b \Gamma_{0}(b)-2 b \Gamma_{1}(b)-\Gamma_{1}(b) \\
& \mathcal{E}_{3}(b)=- \frac{1-\Gamma_{0}(b)+b \Gamma_{1}(b)-b \Gamma_{0}(b)}{1-\Gamma_{0}(b)} \\
& \mathcal{R}_{N G 1}(b)=1-\Gamma_{0}(b)+2 b \Gamma_{0}(b)-2 b \Gamma_{1}(b) \\
& \mathcal{R}_{N G 2}(b)=b\left(\Gamma_{0}(b)-\Gamma_{1}(b)\right) \\
& \mathcal{R}_{\perp \perp 1}^{i}=-\mathcal{R}_{p 2}(b)-\mathcal{R}_{p 3}(b) \\
& \mathcal{R}_{\perp \perp 2}^{i}=\frac{\mathcal{R}_{p 1}(b)}{\Gamma_{0}(b)}+\mathcal{R}_{p 2}(b)\left(\frac{1-\Gamma_{0}(b)}{b\left(\Gamma_{0}(b)-\Gamma_{1}(b)\right)}\right. \\
&\left.\quad+1-\frac{2}{\Gamma_{0}(b)}+\frac{\Gamma_{1}(b)}{\Gamma_{0}(b)}\right) \\
& \quad+\mathcal{R}_{p 3}(b)\left(1-\frac{\Gamma_{0}(b)}{b\left(\Gamma_{0}(b)-\Gamma_{1}(b)\right)}+\frac{\Gamma_{1}(b)}{\Gamma_{0}(b)}\right) \\
& \mathcal{R}_{\perp \perp 3}^{i}=-\frac{\mathcal{R}_{p 1}(b)}{\Gamma_{0}(b)}+\mathcal{R}_{p 2}(b)\left(\frac{2}{\Gamma_{0}(b)}-1-\frac{\Gamma_{1}(b)}{\Gamma_{0}(b)}\right) \\
&-\mathcal{R}_{p 3}(b)\left(1+\frac{\Gamma_{1}(b)}{\Gamma_{0}(b)}\right)
\end{aligned}
$$

where

$$
\begin{aligned}
& \mathcal{R}_{p 1}(b)=4 b^{2} \Gamma_{1}(b)-4 b^{2} \Gamma_{0}(b)-b \Gamma_{1}(b)+3 b \Gamma_{0}(b) \\
& \mathcal{R}_{p 2}(b)=2 b^{2} \Gamma_{1}(b)+b \Gamma_{1}(b)-2 b^{2} \Gamma_{0}(b) \\
& \mathcal{R}_{p 3}(b)=2 b^{2} \Gamma_{0}(b)+2 b^{2} \Gamma_{1}(b)-7 b \Gamma_{1}(b) .
\end{aligned}
$$

The coefficients entering the fourth rank cumulants are

$$
\begin{aligned}
& \mathcal{R}_{\|\| 1}^{r}=\frac{\beta}{2 \mu^{r}} \frac{32-9 \pi}{3 \pi-8} \\
& \mathcal{R}_{\|\| 2}^{r}=-\sqrt{\frac{\beta}{\mu^{r}}} \frac{2 \sqrt{\pi}}{3 \pi-8} \\
& \mathcal{R}_{\| \perp 1}^{r}=2 \times 1.03241 \sqrt{\frac{\beta}{\mu^{r}}} \cos \alpha \\
& \mathcal{R}_{\| \perp 2}^{r}=1.73990 \frac{\beta}{\mu^{r}} \cos \alpha \\
& \mathcal{R}_{\| \perp 3}^{r}=1.73990 \frac{\beta^{2}}{2 \mu^{r}} R_{i}
\end{aligned}
$$

with $\mu^{i}=1$ and $\mu^{e}=m_{e} / m_{i}$, ratio of the electron to ion mass.

Acknowledgements. We thank G. Belmont, F. Califano, V. Génot, P. Hellinger and E. Mjølhus for fruitful discussions. This work was performed in the framework of ISSI team "The effect of ULF turbulence and flow chaotization on plasma energy and mass transfers at the magnetopause" and partially supported by "Programme National Soleil Terre" of CNRS.

Edited by: T. Hada

Reviewed by: E. Mjølhus and another anonymous referee

\section{References}

Baumgärtel, K.: Fluid approach to mirror mode structures, Planetary Space Sc., 49, 1239-1247, 2001.
Baumgärtel, K., Sauer, K., and Dubinin, E.: Towards understanding magnetic holes: Hybrid simulations, Geophys. Res. Lett., 30(14), 1761(1-4), doi:10.1029/2003GL017373, 2003.

Baumgärtel, K., Sauer, K., and Dubinin, E.: Kinetic slow modetype solitons, Nonlinear Proc. Geophys., 12, 291-298, 2005.

Beer, M. A. and Hammett, G. W.: Toroidal gyrofluid equations for simulations of tokamak turbulence, Phys. Plasmas, 3(11), 40464064, 1996.

Brizard, A.: Nonlinear gyrofluid description of turbulent magnetized plasmas, Phys. Fluids B, 4(5), 1213-1228, 1992.

Dorland, W. and Hammett, G. W.: Gyrofluid turbulence models with kinetic effects, Phys. Fluids B, 5(3), 812-935, 1993.

Gary, S. P.: The mirror and ion cyclotron anisotropy instabilites, J. Geophys. Res., 97, (A6), 8519-8529, 1992.

Génot, V., Budnik, E., Jacquey, C., Sauvaud, J., Dandouras, I., and Lucek, E.: Statistical study of mirror mode events in the Earth magnetosheath, AGU Fall Meeting Abstracts, C1412+, 2006.

Goswami, P., Passot, T., and Sulem, P. L.: A Landau fluid model for warm collisionless plasmas, Phys. Plasmas, 12, 102109(19), 2005.

Hall, A.N.: Finite ion Larmor radius modifications to the firehose and mirror instabilities, J. Plasma Physics, 21, 431-443, 1979.

Hau, L. N., Wang, B. J., and Teh, W. L.: Slow mode waves and mirror instability in gyrotropic Hall magnetohydrodynamic model, Phys. Plasmas, 12, 122904(1-8), 2005.

Howes, G. G., Cowley, S. C., Dorland, W., Hammett, G. W., Quataert, E., and Schekochihin, A. A.: Astrophysical gyrokinetics: Basic equations and linear theory, Ap.J., 651, 590-614, 2006.

Joy, S. P., Kivelson, M. G., Walker, R. J., Khurana, K. K., Russell, C. T., and Paterson, W. R.: Mirror mode structures in the Jovian magnetosheath, J. Geophys. Res., 111(A12212), doi:10.1029/2006JA011985, 2006.

Kaufmann, R. L., Horng, J. T., and Wolfe, A.: Large amplitude hydromagnetic waves in the inner magnetosheath, J. Geophys. Res., 75, 4666-4667, 1970.

Kivelson, M. G. and Southwood, D. S.: Mirror instability II: The mechanism of nonlinear saturation, J. Geophys. Res., 101(A8), 17365-17371, 1996.

Kuznetsov, E. A., Passot, T., and Sulem, P. L.: A dynamical model for nonlinear mirror modes near threshold, Phys. Rev. Lett., 98, 235003(1-4), 2007.

Lucek, E.A., Dunlop, M. W., Horbury, T. S., Balogh, A., Brown, P., Cargill, P., Carr, C., Fornacon, K. H., Georgescun, E., and Oddy, T.: Cluster magnetic field observations in the magnetosheath: four-point measurements of mirror structures, Ann. Geophys., 19, 1421-1428, 2001, http://www.ann-geophys.net/19/1421/2001/.

Mjølhus, E.: Velocity-amplitude relationships and polarization in families of MHD solitary waves, Phys. Scr., T122, 135-153, 2006.

Pantellini, P. G. E.: A model of the formation of stable nonpropagating magnetic structures in the solar wind based on the mirror instability, J. Geophys. Res., 103(A3), 4789-4798, 1998.

Passot, T., Ruban, V., and Sulem, P. L.: Fluid description of trains of stationary mirror structures in a magnetized plasma, Phys. Plasmas, 13, 102310(1-10), 2006.

Passot, T. and Sulem, P. L.: A Landau fluid model with finite Larmor radius effects for mirror mode dynamics, J. Geophys. Res., 
111, A04203(1-15), doi:10.1029/2005JA011425, 2006.

Passot, T. and Sulem, P. L.: Stability and formation of mirror structures: a fluid approach, Communications in Nonlinear Science and Numerical Simulation, doi:10.1016/j.cnsns.2007.04.001., in press, 2007.

Passot, T. and Sulem, P. L.: Collisionless magnetohydrodynamics with gyrokinetic effects, Phys. Plasmas, in press, 2007.

Pokhotelov, O. A., Treumann, R. A., Sagdeev, R. Z., Balikhin, M. A., Onishchenko, O. G., Pavlenko, V. P., and Sandberg, I.: Linear theory of the mirror instability in nonMaxwellian space plasmas, J. Geophys. Res., 107(A10), 1312, doi:10.1029/2001JA009125, 2002.

Rönnmark, K.: Waves in homogeneous, anisotropic multicomponent plasmas (WHAMP), Report no 179, Kiruna Geophysical Institute, (1982); Plasma Phys., 25, 699, 1983.

Sahraoui, F., Belmont, G., Rezeau, L., Cornilleau-Wehrlin, N., Pinçon, J. L., and Balogh, A.: Anisotropic turbulent spectra in the terrestrial magnetosheath as seen by the Cluster spacecraft, Phys. Rev. Lett., 96, 075002(1-4), 2006.

Shapiro, V. D. and Shevchenko, V. I.: Quasilinear theory of instability of a plasma with an anisotropic ion velocity distribution, Sov. Phys. JETP, 18, 1109-1116, 1964.
Smolyakov, A. I., Pogutse, I. O., and Hirose A.: Fluid model of collisionless plasma with finite Larmor radius effects, Phys. Plasmas, 2(12), 4451-4454, 1995.

Snyder, P. B., Hammett, G. W., and Dorland, W.: Landau fluid models of collisionless magnetohydrodynamics, Phys. Plasmas, 4, 3974-3985, 1997.

Stasiewicz, K.: Theory and Observations of Slow-Mode Solitons in Space Plasmas, Phys. Rev. Lett., 93, 125004(1-4), 2004a.

Stasiewicz, K.: Reinterpretation of mirror modes as trains of slow magnetosonic soltions, Geophys. Res. Lett., 31, L21804(1-5), doi:10.1029/2004GL021282, 2004b.

Sperveslage, K., Neubauer, F. M., Baumgärtel, K., and Ness, N. F.: Magnetic holes in the solar wind between 0.3 AU and $17 \mathrm{AU}$, Nonlin. Processes Geophys., 7, 191-200, 2000, http://www.nonlin-processes-geophys.net/7/191/2000/.

Southwood, D. J. and Kivelson, M. G.: Mirror instability: 1. Physical mechanism of linear instability, J. Geophys. Res., 98, A6, 9181-9187, 1993.

Sulem, P. L. and Passot, T.: FLR Landau fluids for collisionless plasmas, Communications in Nonlinear Science and Numerical Simulation, doi:10.1016/j.cnsns.2007.05.002., in press, 2007.

Vedenov, A. A. and Sagdeev, R. Z.: Plasma Physics and Problem of Controlled Thermonuclear Reactions, Vol. III, edited by: Leontovich, M. A., English Edition, Pergamon, N.Y., 321-331, 1958. 